\title{
Key concerns in terms of the response to the COVDD-19 pandemic
}

Weifeng Shen ${ }^{1,2}$

\footnotetext{
${ }^{1}$ Department of Emergency Medicine, the Second Affiliated Hospital, Zhejiang University School of Medicine, Hangzhou, China ${ }^{2}$ Institute of Emergency Medicine, Zhejiang University, Hangzhou, China
}

At present, there are great challenges to the effective control and medical treatment of COVID-19. Emerging and important issues need to be considered in the response to COVID-19 to avert an even greater public health crisis.
$\mathrm{T}$ here are great challenges to the effective control and medical treatment of coronavirus disease 2019 (COVID-19). Ten important issues need to be considered in the response to COVID-19 to avert an even greater public health crisis.

\section{TEN IMPORTANT ISSUES}

First, areas with severe outbreaks have generally received the most attention. However, vulnerable links of health care systems with specific populations may remain unaddressed because of reasons such as detection capabilities. High-risk places, such as hospitals, prisons, and nursing facilities, with clusters of diseased individuals require close attention.

Second, infectious disease models estimating the peak of the pandemic should not be used as simplistic prediction models. Subsequent waves of COVID-19 will likely emerge, and preparations are needed for a long-term response. It is important to maintain an effective balance between minimizing the spread of the disease; maintaining social and economic operations; and meeting basic needs, such as food security, as far as possible.

Third, as the emergency reserves and external support materials for disease control and patient treatment are currently low and temporary [1], supplies should be stockpiled for a long-term pandemic. The production capacity of pandemic-related public health and medical materials should be accelerated and the supply chain strengthened.

Fourth, water is the key substance on which people depend for survival. It is important to be alert to potential pollution of water sources and water supply systems by the virus causing COVID-19, that is, severe acute respiratory syndrome coronavirus 2 (SARS-CoV-2). Comprehensive monitoring systems and treatment facilities are needed to monitor and protect water supply systems.

Fifth, there is little doubt that many cases of COVID-19 remain undetected. Infected but asymptomatic persons may remain unscreened because of a shortage of tests or because of their own reluctance [2]. Obtaining accurate prevalence data requires sufficient numbers of reliable test kits, their wide-spread distribution, and large-scale screening capacity and infrastructure [3]. Under the premise of sufficient nucleic acid detection capability, large-scale screening of specific high-risk populations can be considered. Through the widespread use of screening tests, the high proportion of positive persons in the screening population should be more alarming than the increase in the number of persons testing positive. 
Ten important issues need to be considered in the response to COVID-19. These key issues are vulnerable links of health care systems, preparations for a long-term response, the production capacity of pandemic-related public health and medical materials, comprehensive monitoring systems and treatment facilities needed to monitor and protect water supply systems, large-scale screening of specific high-risk populations, a wide range of pre-inspection network, establishment of a multi-level epidemic prevention and control system, access to medical resources for COVID-19 patients, preventing infection of front-line medical workers and the implementation of multi-level cooperation.

Sixth, during a pandemic, pre-examination in fever clinics [4], out-patient wards, and emergency medical services is critical. Also necessary is a wide range of pre-inspection practices at road checkpoints, airport terminals, border crossings, and public places with a large inflow of people. Both systems must be woven into a prevention and control network.

Seventh, establishment of a multi-level epidemic prevention and control barrier is fundamental for public protection. At the societal level, joint prevention and control mechanisms can effectively improve organizational capabilities. Community prevention and control is the basis of multi-level epidemic management and control [5]. During rapid disease spread, regardless of whether there is community transmission, community "grid" prevention and control can play the role of a community "gatekeeper." Digital tracking of the entire population also improves the efficiency of epidemic prevention and disease control [6], but it has limitations.

Eighth, the lack of access to medical resources for COVID-19 patients contributes to the spread of the disease, and every effort should be made to go beyond testing, to admission and treatment. Municipalities should have protocols and the capacity for rapidly upscaling the building and renovation of hospitals. "Re-purposing" existing structures, such as conversion of public venues such as stadiums and exhibition centers into "Fangcang" shelter hospitals in China, can be an effective method of expanding health care capacity [7]. Nosocomial cross-infection is an important pathway for the transmission of COVID-19 [8], and the "three preventions" - preventing cross-infection among patients, patient escorts, and medical staff - must be implemented in all facilities.

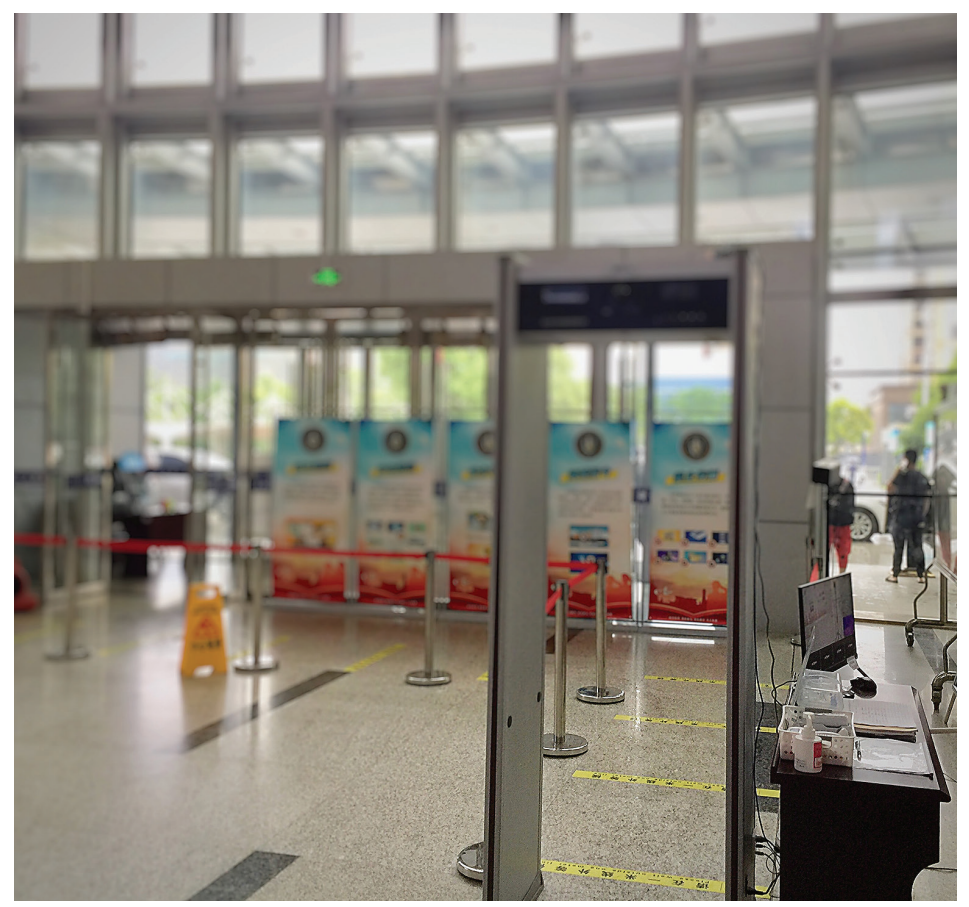

Photo: Automatic temperature measurement of personnel entering the hospital (from the author's own collection, used with permission).
Ninth, in the situation of shortage of effective personal protective equipment, preventing infection of front-line medical workers is a priority. Measures such as staff rotation and psychological support can help protect these key personnel. Psychological needs of COVID-19 patients and families in isolated areas must also be addressed.

Tenth, regional differences in the implementation of major public health emergency measures, such as suspending intra-city public transport and school closures $[9,10]$, have become more apparent. These differences make many effective measures difficult to achieve a large-scale impact. The implementation of multi-level cooperation, including internationally, needs to be greatly improved.

\section{CONCLUSION}

In short, ten important issues mentioned above need to be considered in the response to COVID-19. During the development of the COVID-19 epidemic, we still need to find more issues in time and respond actively. 
Acknowledgements: The author would like to thank Quzhou Branch of the Second Affiliated Hospital, Zhejiang University School of Medicine.

Funding: None.

Authorship contributions: WS contributed to the conception of the study and wrote the manuscript.

Competing interests: The author completed the The ICMJE Unified Competing Interest form (available upon request from the corresponding author), and declare no conflicts of interest.

1 Remuzzi A, Remuzzi G. COVID-19 and Italy: what next? Lancet. 2020;395:1225-8. Medline:32178769 doi:10.1016/ S0140-6736(20)30627-9

2 Qiu J. Covert coronavirus infections could be seeding new outbreaks. Nature. 2020; Online ahead of print. Medline:32203376 doi:10.1038/d41586-020-00822-x

3 Gudbjartsson DF, Helgason A, Jonsson H, Magnusson OT, Melsted P, Norddahl GL, et al. Spread of SARS-CoV-2 in the Icelandic population. N Engl J Med. 2020; Online ahead of print. Medline:32289214 doi:10.1056/NEJMoa2006100

4 Zhang J, Zhou L, Yang Y, Peng W, Wang W, Chen X. Therapeutic and triage strategies for 2019 novel coronavirus disease in fever clinics. Lancet Respir Med. 2020;8:e11-2. Medline:32061335 doi:10.1016/S2213-2600(20)30071-0

5 Pan A, Liu L, Wang C, Guo H, Hao X, Wang Q, et al. Association of public health interventions with the epidemiology of the COVID-19 outbreak in Wuhan, China. JAMA. 2020; Online ahead of print. Medline:32275295 doi:10.1001/ jama.2020.6130

6 Ferretti L, Wymant C, Kendall M, Zhao L, Nurtay A, Abeler-Dörner L, et al. Quantifying SARS-CoV-2 transmission suggests epidemic control with digital contact tracing. Science. 2020; Online ahead of print. Medline:32234805 doi:10.1126/ science.abb6936

7 Chen S, Zhang Z, Yang J, Wang J, Zhai X, Bärnighausen T, et al. Fangcang shelter hospitals: a novel concept for responding to public health emergencies. Lancet. 2020;395:1305-14. Medline:32247320 doi:10.1016/S01406736(20)30744-3

8 Wang Z, Wang J, He J. Active and effective measures for the care of patients with cancer during the COVID-19 spread in China. JAMA Oncol. 2020; Online ahead of print. Medline:32236504 doi:10.1001/jamaoncol.2020.1198

9 Tian H, Liu Y, Li Y, Wu C, Chen B, Kraemer MUG, et al. An investigation of transmission control measures during the first 50 days of the COVID-19 epidemic in China. Science. 2020; Online ahead of print. Medline:32234804 doi:10.1126/ science.abb6105

10 Koo JR, Cook AR, Park M, Sun Y, Sun H, Lim JT, et al. Interventions to mitigate early spread of SARS-CoV-2 in Singapore: a modelling study. Lancet Infect Dis. 2020; Online ahead of print. Medline:32213332 doi:10.1016/S14733099(20)30162-6

\section{Correspondence to:}

Weifeng Shen

Department of Emergency Medicine, the Second

Affiliated Hospital

Zhejiang University School of Medicine

Institute of Emergency Medicine

Zhejiang University

88\# Jiefang road, Shangcheng District

Hangzhou, 310009

China

2199023@zju.edu.cn 